\title{
FAUNA DE VERTEBRADOS DEL CRETÁCICO INFERIOR DEL YACIMIENTO DE “ZORRALBO” EN GOLMAYO (SORIA, ES- PAÑA)
}

\author{
Carolina FUENTES VIDARTE, Manuel MEIJIDE \\ CALVO, Federico MEIJIDE FUENTES \& Manuel \\ MEIJIDE FUENTES
}

C/ Almazán 17, 2 C. 42004 Soria. carolfuentes@usuarios.retecal.es

\begin{abstract}
Fuentes Vidarte, C., Meijide Calvo, M., Meijide Fuentes, F. \& Meijide Fuentes, M. 2005. Fauna de vertebrados del Cretácico Inferior del Yacimiento de "Zorralbo" en Golmayo (Soria, España). [Vertebrate fauna (Lower Cretaceous) of the site "Zorralbo" a Golmayo (Soria, Spain).] Revista Española de Paleontología, N.E. X, 83-92. ISSN 0213-6937.
\end{abstract}

\begin{abstract}
A new locality of Early Cretaceous (late Hauterivian/early Barremian) age near Golmayo (Soria, Spain) has yielded a varied vertebrate assemblage consisting of five dinosaurs taxa remains (Ornithopoda, Sauropoda, Theropoda, and Thyreophora), a Cryptodiran turtle and a "Mesosuchian" crocodilian.

This fauna provides new evidence about the composition of Lower Cretaceous European ecosystems. By the diversity of its fauna, the "Zorralbo" site provides an important contribution to our knowledge of the Early Cretaceous vertebrates of Spain.
\end{abstract}

Keywords: Early Cretaceous, Soria (Spain), Dinosauria, Chelonii, Crocodyliformes.

\section{RESUMEN}

El yacimiento de "Zorralbo" en el municipio de Golmayo (Soria, España), perteneciente al Cretácico Inferior (Hauteriviense superior/Barremiense inferior), ha proporcionado un conjunto faunístico formado por los restos de cinco taxones de dinosaurios (Ornithopoda, Sauropoda, Theropoda y Thyreophora), una tortuga Criptodira y un cocodrilo "Mesosuquio", fauna que nos ayuda a comprender la composición de los ecosistemas europeos en el Cretácico Inferior y que constituye una aportación importante al conocimiento de los vertebrados del Cretácico Inferior de España.

Palabras clave: Cretácico Inferior, Soria (España), Dinosauria, Chelonii, Crocodyliformes.

\section{INTRODUCCIÓN}

Hasta ahora, la provincia de Soria había proporcionado muy pocos fósiles directos de vertebrados Mesozoicos:

Del Cretácico inferior, concretamente del tránsito Titónico-Berriasiense medio se han citado unos pocos restos de pterosaurios recuperados en la sierra de Oncala (Fuentes Vidarte \& Meijide Calvo, 1996). Del Hauteriviense superior-Barremiense inferior son los escasos restos de tortugas, cocodrilos, peces y dinosaurios hipsilofodontidos del yacimiento de "Los Caños" en Golmayo (Royo y Gómez, 1926a, 1926b, 1926d; Saenz García 1932, 1955; Buscalioni, 1986; Fuentes Vidarte, 1988; Sanz et al., 1992; Sanz \& Moratalla, 1993; Fuentes Vidarte et al., 2003). En 1982 se encontró en el pueblo de Tera un fémur de un gran dinosaurio saurópodo, posiblemente Diplodocoideo, que se conserva en el Museo Numantino de Soria, aún sin publicar (Sanz et al., 1992), adscrito al Cretácico Inferior.

En el Cretácico superior del pueblo de Cubilla (Mastrichtiense) aparecieron restos autopodiales atribuidos al género Rhabdodon y una vértebra que se clasifico como perteneciente al saurópodo Hypselosaurus (Lapparent et al., 1957) y que posteriormente se ha clasificado como Titanosauria indet. (Pereda Suberbiola \& Ruiz-Omeñaca, 2001).

A principios del año 2000, durante una prospección realizada siguiendo la denominada franja Wealdense (Palacios \& Sánchez Lozano, 1885; Palacios, 1890; Alía Medina, 1942) que discurre al oeste de Soria capital, en las 
cercanías del pueblo de Golmayo, encontramos en superficie varios restos fósiles que atribuimos desde el primer momento a un dinosaurio.

Vista la importancia del hallazgo y solicitados los permisos pertinentes a la Dirección General de Patrimonio de la Junta de Castilla y León, se realizó una cata de urgencia en la que se extrajeron los restos de un ejemplar de dinosaurio "iguanodóntido".

En el año 2001 se encontraron no solo nuevos restos de este dinosaurio, sino también los de un dinosaurio saurópodo de gran tamaño; en el 2002 se extrajeron nuevos restos de los dinosaurios citados y numerosos osteodermos y espinas de un dinosaurio acorazado, y en el 2003, los primeros restos de un dinosaurio terópodo de mediano-gran tamaño.

A lo largo de las cuatro campañas de excavación se han obtenido además restos de otros vertebrados (un dinosaurio hipsilofodóntido, cocodrilos, tortugas), de invertebrados (moldes internos de lamelibranquios del género Unio) y restos de coníferas.

Todos los restos obtenidos han sido inventariados y siglados (más de 4.000 piezas), un alto porcentaje de los mismos ha sido restaurado y el conjunto se encuentra depositado en el Museo Numantino de Soria.

\section{CONTEXTO GEOGRÁFICO-GEOLÓGICO}

El yacimiento de "Zorralbo" se localiza en el paraje del mismo nombre, en el término municipal de Golmayo, a 5 $\mathrm{km}$ al oeste de Soria capital. Sus coordenadas UTM, según el Mapa Topográfico Nacional, hoja 342, escala 1:50.000 son: 30TWM394238.

Geológicamente el yacimiento queda incluido en la subcuenca occidental de la gran Cuenca sedimentaria de Cameros, Grupo Tera (Tischer, 1966; Beuther, 1966), en el Sector de Soria (Área de Soria, según Clemente \& Pérez Arlucea, 1993) y, dentro de éste, en la Formación Golmayo (Clemente, 1988; Clemente \& Alonso, 1990).

De las cinco Secuencias Deposicionales desarrolladas en la Cuenca de Cameros occidental, la Formación Golmayo pertenece a la S. D. III (Mas et al., 1993), con una edad Hauteriviense superior/Barremiense inferior ligeramente más antigua que la del resto de las formaciones de esta subcuenca, según parece indicar su asociación carofítica (Martín-Closas \& Alonso Millán, 1998).

La Formación Golmayo se extiende desde las estribaciones orientales de la Sierra de Cabrejas, de edad Cretácico Superior, hasta la Sierra de San Marcos, de la misma edad y desde el oeste de la ciudad de Soria hasta la falla de las Cuevas de Soria, que la corta en dirección NE-SO. Estratigráficamente se desconoce su base debido a la falla ya mencionada (Meléndez, 1978; Clemente \& Alonso, 1990) mientras que sobre su techo se apoya la Formación Pantano de la Cuerda del Pozo, perteneciente al Barremiense medio (Clemente et al., 1991).
La Formación Golmayo es una unidad compleja y heterogénea, formada por aproximadamente $700 \mathrm{~m}$ de areniscas en cuerpos canalizados, calizas y margas y, de modo subordinado y hacia techo, por conglomerados silíceos y calcáreos. La arquitectura deposicional de la Formación parece indicar que se originó en una zona con una elevada tasa de subsidencia, relacionada con el funcionamiento de la falla de Las Fraguas (Clemente \& Alonso, 1990).

El yacimiento de "Zorralbo" está formado por dos estratos de arenisca y un estrato intermedio arcilloso que se extienden en dirección $30^{\circ} \mathrm{NE}$. El techo tiene un buzamiento de $85^{\circ}$ con respecto a la horizontal; el suelo buza entre $75^{\circ} \mathrm{y}$ $85^{\circ}$ con respecto a la horizontal; el estrato intermedio (que es en donde aparecen los restos fósiles) está formado por lutitas, margas y areniscas de colores verdosos y rojizos, que configuran una masa de gran dureza conocida en la región como "piedra nuez". Hasta el momento y después de cuatro campañas de excavación (2000-2003), el área excavada tiene una profundidad media de $10 \mathrm{~m}$ y $15 \mathrm{~m}$ de longitud siendo su potencia variable entre 70 y $120 \mathrm{~cm}$.

El ambiente en que se habría originado la Formación Golmayo sería, según Clemente (1988) y Clemente \& Alonso (1990), "el de un sistema fluvio-lacustre con varios sub-ambientes: canales principales muy sinuosos, llanura de inundación, lagos, charcas, derrames laterales, pequeños canales de drenaje y zonas de vegetación".

\section{MATERIAL Y MÉTODOS}

En primer lugar se procedió a recoger el material fósil aparecido en superficie, que cubría una extensión aproximada de $150 \mathrm{~m}^{2}$ y a continuación se abrió una cata de aproximadamente $5 \times 5 \mathrm{~m}$ en la zona donde había aparecido uno de los restos de mayor tamaño, una escápula, semienterrado. Así se comprobó que la zona fértil era muy reducida y que se encontraba entre dos estratos de arenisca que sobresalían en el terreno.

La zona de excavación se ha dividido en cuadrículas de 1x1 m tomando como uno de sus límites el techo del yacimiento (15 cuadrículas). Dadas las dificultades que entraña esta excavación se ha tenido que establecer una zona escalonada de protección a su alrededor, de unos $25 \times 20 \mathrm{~m}$ de diámetro, con una vía de acceso de 2,5 $\mathrm{m}$ de anchura en forma de rampa para extracción del derrubio. Se ha despejado una zona baldía donde acumular ese derrubio, que se ha examinado y cribado constantemente, dado que según la Dirección General de Patrimonio de la Junta de Castilla y León, deberá servir para rellenar la zona de excavación cundo esta termine.

Los restos fósiles una vez descubiertos contrastan claramente con el sedimento y pueden ser extraídos del mismo con cierta facilidad empleando medios convencionales aunque para abrir la zona de excavación es necesario trabajar con un martillo neumático; en diversas ocasiones hubo que proceder a una consolidación in situ debido a su mal estado por exceso de humedad (cuando llueve se produce una fuerte escorrentía en todo el terreno), con cianocrilatos muy fluidos y de pegado rápido o al escayolado para facilitar su transporte hasta la superficie. 
Al mismo tiempo se ha ido levantando el plano del área excavada y de la posición relativa de los restos que iban apareciendo.

El material recogido se ha inventariado según la numeración proporcionada por el Museo Numantino de Soria:

Año 2000: sigla 2000/132 (1340 restos)

Año 2001: sigla 2001/122 (1520 restos)

Año 2002: sigla 2002/95 (870 restos)

Año 2003: sigla 2003/69 (505 restos)

De todos los restos extraídos hasta el momento, el $65 \%$ puede adscribirse a un animal determinado y el $35 \%$ restante se refiere a esquirlas y fragmentos indeterminables.

\section{DATOS TAFONÓMICOS}

Dado que aún no se ha cerrado la excavación, no podemos elaborar un análisis tafonómico completo, por lo que en esta publicación tan solo damos unos datos generales, sin intentar profundizar en los mismos.

El yacimiento ha proporcionado una rica asociación de vertebrados continentales, ya que hasta el momento se han extraído los restos de cinco taxones de dinosaurios (Ornithopoda, Sauropoda, Theropoda y Thyreophora), de un cocodrilo "Mesosuquio"y de una tortuga Cryptodira.

También han aparecido moldes internos de bivalvos dulceacuícolas (Unio sp.), restos vegetales carbonizados y numerosos coprolitos sin atribución.

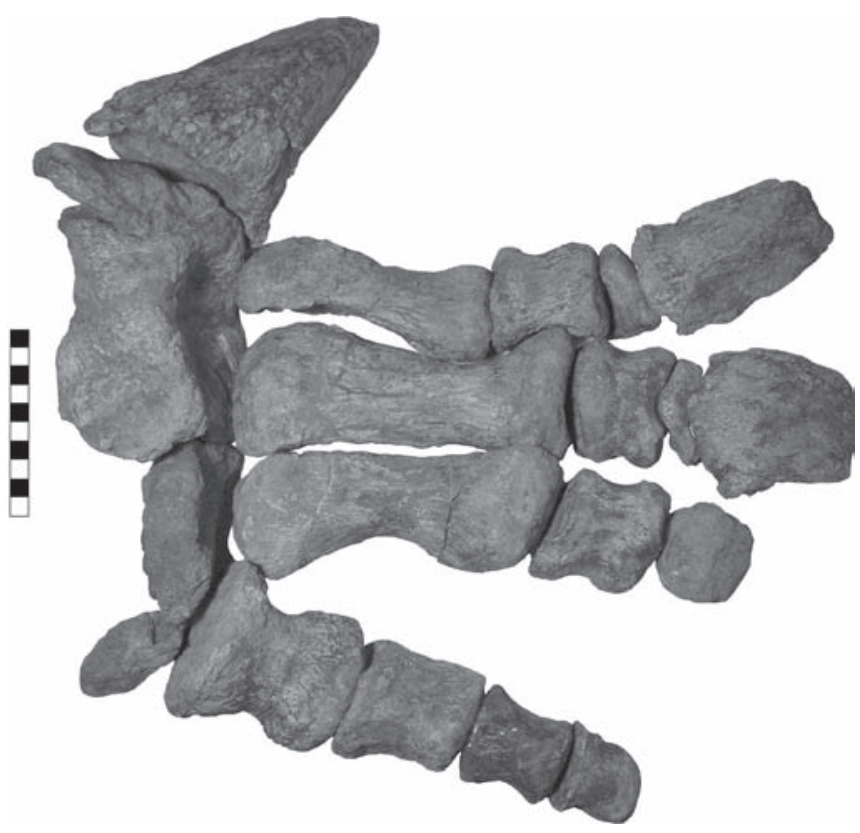

Figura 1. Mano derecha en vista dorsal de Iguanodon sp. del yacimiento de "Zorralbo", Golmayo (Soria, España). M. N. S. 2000/132.1049 a 2000/132.1063 y 2000/132.100 a 2000/132.101. Escala $=10 \mathrm{~cm}$.

Right manus in dorsal aspect of Iguanodon sp. from "Zorralbo" site a Golmayo (Soria, Spain). M. N. S. 2000/132.149 to 2000/132.1063 and 2000/132.1002000/132.101. Scale bar $=10 \mathrm{~cm}$.
La asociación fósil es un conjunto heterogéneo formado por una mezcla de huesos de gran tamaño, dientes aislados, porciones esqueléticas articuladas (mano de un dinosaurio "iguanodóntido", vértebras sacras de un dinosaurio terópodo) o en relación anatómica (cintura pelviana de un dinosaurio saurópodo).

La conservación de los restos es diversa, desde elementos con una conservación excelente como la extremidad anterior de un ejemplar del género Iguanodon (Fig. 1), espinas de un ejemplar del género Polacanthus (Fig. 2), dientes diversos, hasta fragmentos óseos muy redondeados por la abrasión pero en los que aún se reconocen algunas estructuras que permiten su asignación (estadío 2 de Fiorillo, 1988).

El grado de integridad de los restos fósiles es muy variable, con una importante proporción de fragmentos de menos de $3 \mathrm{~cm}$ de longitud y numerosas esquirlas aún menores que corresponden, en gran parte, al sistema trabecular del interior de las vértebras de un dinosaurio saurópodo. Las fracturas son fundamentalmente transversales al hueso, de bordes limpios, en muy pocos casos astillados. Las fracturas longitudinales son muy escasas

En los fósiles no se aprecia ninguna actividad de depredación, no aparecen huesos con bordes aserrados que como dice Brain (1981) deben atribuirse a los mordiscos de predadores, ni tampoco corrosión química por ácidos
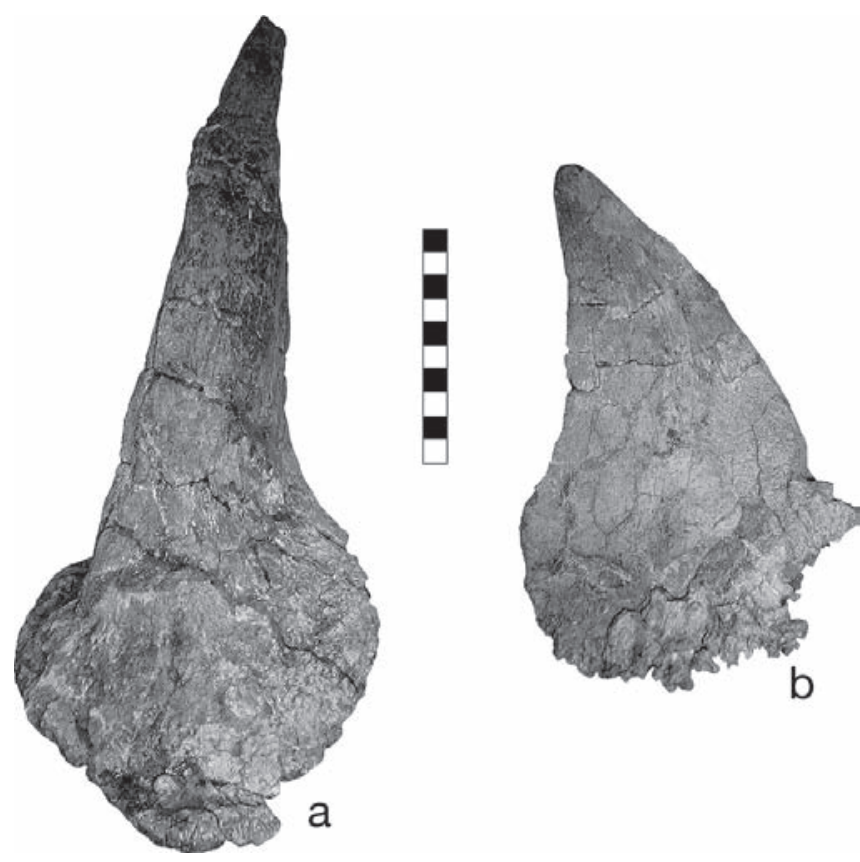

Figura 2. Yacimiento de "Zorralbo", Golmayo (Soria, España). a, Espina presacra izquierda de Polacanthus sp. M. N. S. 2003/69.479. b, Espina caudal anterior derecha de $\mathrm{Po}$ lacanthus sp. M. N. S. 2002/95.117. Escala $=10 \mathrm{~cm}$. "Zorralbo" site a Golmayo (Soria, Spain). a, Left presacral spine of Polacanthus sp. M. N. S. 2003/69.479. b, Right anterior caudal spine of Polacanthus sp. M. N. S. 2002/95.117. Scale bar $=10 \mathrm{~cm}$. 
húmicos (Fiorillo, 1988), aunque algunos restos de dientes de Iguanodon presentan pequeñas perforaciones en el esmalte que podrían ser atribuidas a la acción de los jugos gástricos del propio dinosaurio (Metcalf, 1993).

Una importante proporción de los restos aparece recubierta por óxidos de hierro y al menos el $10 \%$ de los fragmentos óseos está carbonizado mientras que otro $10 \%$ ha sido deformado por una falla que recorre el yacimiento en toda su longitud.

\section{PALEONTOLOGÍA SISTEMÁTICA}

\author{
Clase DINOSAURIA Bakker \& Galton, 1974 \\ Orden ORNITHISCHIA Seeley, 1888 \\ Suborden CERAPODA Sereno, 1986 \\ Infraorden ORNITHOPODA Marsh, 1881 \\ Familia Iguanodontidae Cope, 1869 \\ Genus Iguanodon Mantell, 1825
}

\section{Iguanodon sp.}

Fig. 1

Este grupo está bien representado por los restos de un ejemplar de Iguanodon sp., del que se han recuperado fragmentos del maxilar y de la mandíbula, la cintura escapular completa, la extremidad anterior derecha y el húmero izquierdo, fragmentos de los diferentes componentes de la cintura pelviana, del sacro y de la extremidad posterior derecha, así como 35 cuerpos vertebrales (dorsales y caudales), 60 fragmentos de costillas casi completas (cervicales, dorsales y caudales) y numerosos fragmentos de tendones osificados.

También, se han recuperado 175 dientes en uso, muy gastados, 3 dientes de reposición y otros tantos gérmenes del mismo taxón.

Estos restos fósiles se atribuyen al género Iguanodon siguiendo las descripciones de Norman $(1980,1986)$ y debido a:

La existencia de un gran foramen en el hueso suprangular, inexistente en los Hadrosauridae (Weishampel \& Horner, 1992) pero presente en los Iguanodontidae, géneros Iguanodon y Ouranosaurus Taquet, 1976.

A la morfología de sus dientes, recubiertos de esmalte por su cara lingual (mandibular) o por la labial (maxilar). En los dientes inferiores la cara lingual presenta dos cretas, la primaria más fuerte y siempre posterior a la secundaria; en los dientes superiores, la cara labial presenta una crestas en posición subcentral. Es decir, su morfología es la típica del género Iguanodon e indistinguible de la del género Ouranosaurus, pero este género nunca se ha citado fuera de su lugar de aparición (Nigeria) y está muy alejado en el tiempo de los fósiles de "Zorralbo", ya que pertenece al Aptiense superior.

A la presencia de cinco dedos funcionales en la mano, el primero transformado en un fuerte espolón, con la pri- mera falange reducida a un estrecho disco. La fórmula falangeal de la mano es 2-3-3-2-3 o 4 . El primero y el quinto dedo se separan del eje del brazo; las falanges ungueales del segundo y tercer dedo están transformadas en pezuñas; el quinto dedo es el más fino y largo (Fig. 1). En Hadrosauridae, la mano tiene solo cuatro dedos y estos tienen tres falanges.

Y por último, por la existencia de protoatlas, determinado por Dollo (1885) en Iguanodon y por Taquet (1976) en Ouranosaurus aunque muy diferentes en los dos géneros; por que el proceso prepúbico es más estrecho transversalmente que dorsalmente (característica del género Iguanodon) y por la presencia de tendones osificados de dos tipos.

Dadas las diferencias anatómicas y morfométricas observadas al comparar estos restos con los de las especies asignadas al género Iguanodon, creemos que el ejemplar de "Zorralbo" podría tratarse de una nueva especie.

Familia Hypsilophodontidae Dollo, 1882.

\section{Hypsilophodontidae indet.}

La presencia de un representante de esta familia es vestigial, solamente se han recuperado tres centros de vértebras caudales sin procesos transversos (Galton, 1974). Los centros son anficélicos, de caras articulares ligeramente cóncavas y caras laterales que se comprimen en el centro. La cara ventral es cóncava y presenta las correspondientes facetas para los chevrones y en la cara dorsal son muy visibles las suturas casi dendrítica para los pedicelos del arco neural.

Las características morfológicas y morfométricas de los restos estudiados están dentro de las dadas por Galton (1974) para la especie tipo Hypsilophodon foxii Huxley, 1869 pero, no se han encontrado elementos suficientes para su caracterización como son los dientes, la escápula, la cintura pelviana o el fémur.

\section{Suborden ANKYLOSAURIA Osborn, 1923 \\ Familia Nodosauridae Marsh, 1890 \\ Genus Polacanthus Owen, 1865}

\section{Polacanthus sp.}

Fig. 2

En el caso de los dinosaurios acorazados se han recogido numerosos osteodermos o placas dérmicas relativamente macizas, de bordes redondeados y formadas por un entramado de fibras visible a simple vista, con formas muy variables, desde simples discos ovales o redondeados a discos ovales asimétricos con una quilla o una espina dorsal y placas rectangulares ligeramente convexas y sin ningún tipo de quilla.

Se han recuperado también tres espinas presacras y 11 espinas caudales más o menos triangulares, comprimidas lateralmente y de base hueca (Fig. 2) y parte del sacro y del 
ileon derecho, los centros y parte del arco neural de cuatro vértebras dorsales y varios fragmentos de costillas.

Este material se ha asignado al género Polacanthus tomando como base la revisión de dicho género realizada por Pereda Suberbiola (1994).

Orden SAURISCHIA Seeley, 1888

Suborden SAUROPODOMORPHA Huene, 1932

Infraorden SAUROPODA Marsh, 1878

Familia Brachiosauridae Riggs, 1904

\section{Brachiosauridae indet.}

Fig. 3

Los restos extraídos hasta el momento pertenecen a un solo ejemplar del que se han obtenido el húmero y el fémur derechos, parte de la cintura pelviana (pubis e isquion derechos), tres vértebras dorsales muy fragmentadas y dos caudales completas, fragmentos de costillas dorsales y caudales y falanges ungueales de la mano.

En el húmero el extremo proximal está bien desarrollado y muy ensanchado en sentido transverso; la cresta deltopectoral es muy patente, llegando hasta la mitad de la longitud del hueso; la diáfisis tienen sección elíptica, con el eje mayor en sentido transverso y de aproximadamente doble longitud que el eje anteroposterior, el extremo distal está ensanchado transversalmente, con ecto y endoepicóndilos poco marcados y un pequeño canal anterior que los separa y en el que sobresale una pequeña cresta (Fig. 3).

La longitud total del húmero es de $135 \mathrm{~cm}$; su diámetro proximal es de $36 \mathrm{~cm}$, el distal es de $38 \mathrm{~cm}$ y el diámetro transverso mínimo de la diáfisis es de $18 \mathrm{~cm}$. Aplicando la fórmula:

$\mathrm{Ig}=$ diám. transv. proximal + diam. transv. medio + diám. transv. distal/longitud el índice de gracilidad resultante es igual a 68, lo que le acerca a Brachiosaurus brancai Janensch, 1914, cuyo índice es de 63 y a Botrhiospondylus madagascarensis Lydekker, 1895 con un índice de 71 y al Titanosauriforme de Morella (Castellón), con índice de 72 (datos de Santafé et al., 1982).

El fémur está casi completo, falta parte de la extremidad proximal que en la figura 3 aparece restaurado. La diáfisis es muy ancha por encima del cuarto trocanter y se estrecha progresivamente por debajo del mismo para volver a ensancharse ligeramente por encima de los cóndilos distales; en el contorno proximal se marca una comba muy patente; el extremo distal presenta un par de cóndilos muy desarrollados y asimétricos, separados por un canal poco profundo. La longitud estimada es de $150 \mathrm{~cm}$.

La relación longitud del fémur/longitud del húmero es de 1,1; en el Titanosauriforme de Morella (Castellón) esa relación es de 1 (Santafé et al., 1982).

Las vértebras dorsales poseen amplios pleuroceles y un centrum muy pneumatizado, formado por celdillas mili y
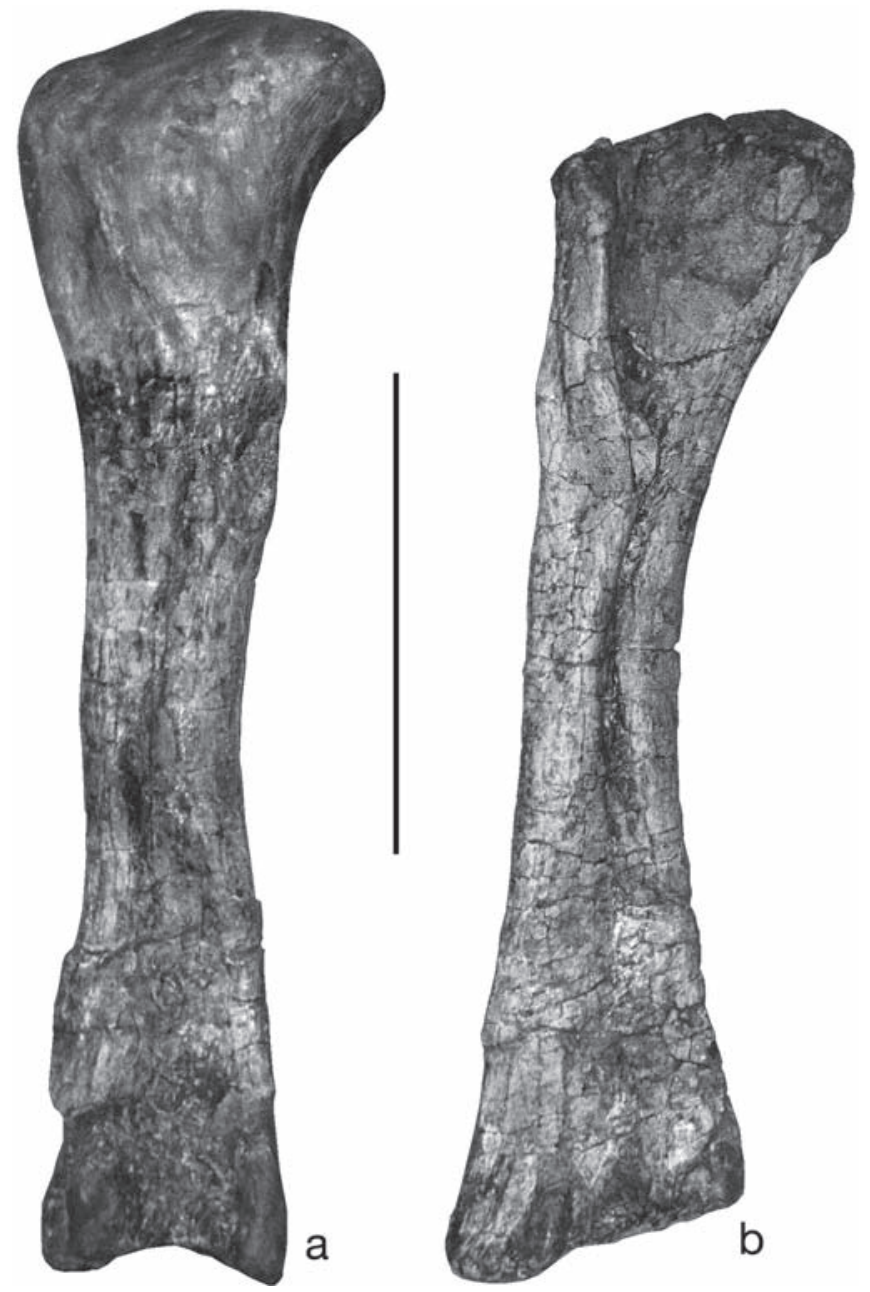

Figura 3. Yacimiento de "Zorralbo", Golmayo (Soria, España). Brachiosauridae indet. a, Fémur derecho de un dinosaurio saurópodo. Vista de la cara anterior. M. N. S. 2001/122.611. b, Húmero derecho de un dinosaurio saurópodo (Saurischia, Brachiosauridae). Vista de la cara interior. M. N. S. 2001/122.613. Escala $=50 \mathrm{~cm}$. "Zorralbo" site a Golmayo (Soria, Spain). Brachiosauridae indet. a, Rightfemur, anterior wiew. Sauropod dinosaur.M.N.S.2001/122.611.b, Righthumerus, interior wiew. Sauropod dinosaur (Ornithischia, Brachiosauridae). M. N.S. 2001/122.613. Scale bar $=50 \mathrm{~cm}$.

centimétricas; las costillas caudales tienen forma de $\mathrm{Y}$ en norma anterior y de $\mathrm{V}$ en norma posterior.

Con los datos expuestos creemos que los restos del saurópodo de "Zorralbo" deberían incluirse en el grupo de los Titanosauriformes (Salgado et al., 1997; Wilson \& Sereno, 1998) y dentro de éstos, en la familia Brachiosauridae.

Orden SAURISCHIA Seeley, 1888 Suborden THEROPODA Marsh, 1881

THEROPODA indet.

Fig. 4 
Los grandes dinosaurios carnívoros están representados por varios dientes curvados hacia atrás, comprimidos labiolingualmente, con carenas mesial y distal denticuladas y sección basal elíptica. No se ha conservado ninguna raíz y la altura de la corona varía entre 2 y $8 \mathrm{~cm}$. El esmalte cubre el diente uniformemente y es liso.

Se han obtenido también cinco vértebras sacras (Fig. 4), procélicas, dos de ellas fusionadas y dos vértebras dorsales, que se caracterizan por la presencia de una quilla subcentral en la cara ventral del centrum y la conocida ornamentación de crestas y surcos que bordean las caras articulares anterior y posterior del suborden Theropoda. Se han extraido dos costillas cervicales, varios fragmentos de costillas dorsales y restos de chevrones.

Clase REPTILIA Laurenti, 1768 Orden CHELONII Brongniart, 1800

\section{CHELONII indet.}

El grupo de los Quelonios está representado por varias placas aisladas del caparazón y una vértebra caudal. La ornamentación de las placas está formada por granulaciones que en ocasiones se unen formando cortas vermiculaciones poco marcadas

Orden CROCODYLIA Gmelin, 1878

Suborden MESOSUCHIA Huxley, 1875

Familia Goniopholidae Cope, 1875

\section{Goniophilidae indet}

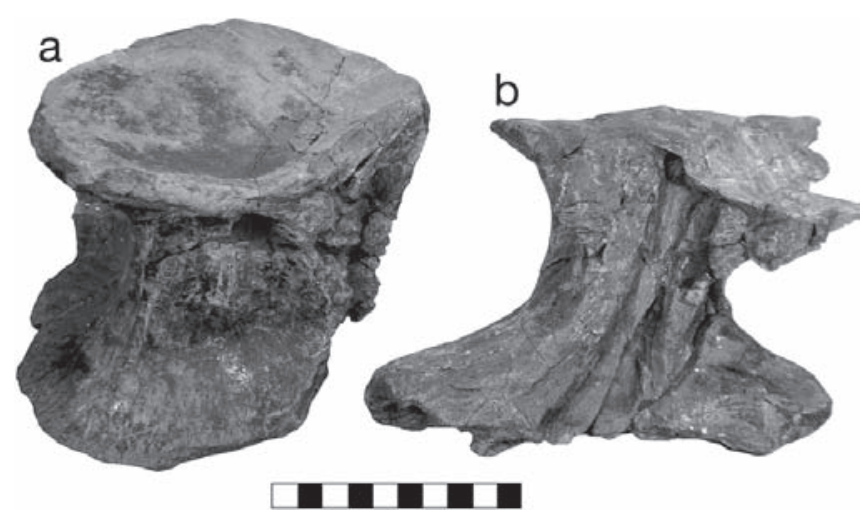

Figura 4. Yacimiento de "Zorralbo", Golmayo (Soria, España). a, Centrum de vértebra sacra en norma lateral de Theropoda indet. M. N. S. 2003/69.431. b, Centrum de vértebra sacra en norma dorsal de un dinosaurio terópodo (Saurischia, Theropoda indet.). M. N. S. 2003/69.431. Escala $=10 \mathrm{~cm}$.

"Zorralbo" site a Golmayo (Soria, Spain). a, sacral centrum in lateral aspect of Theropoda indet. M. N. S. 2003/69.431. b, Sacral centrum in dorsal aspect. Theropod dinosaur (Saurischia, Theropoda indet.). M. N. S. 2003/69.431. Scale bar $=10 \mathrm{~cm}$.
Este grupo está representado en "Zorralbo" por dientes aislados, cónicos, puntiagudos, curvados hacia atrás, con una arista mesial y otra distal bien visibles, de sección transversa ovalada y ornamentación formada por finas estrías excavadas en la corona, siendo la altura de la corona variable entre 0,5 y $6 \mathrm{~cm}$.

Se han encontrado también varias vértebras caudales y dos osteodermos más o menos rectangulares, de superficie alveolada.

Se atribuyen a un representante de la familia Goniopholidae, ya citada en Soria (Royo y Gómez, 1926d, 1929).

Se ha recuperado también un fragmento de maxilar derecho de muy pequeño tamaño $(4 \mathrm{~cm})$, actualmente en estudio.

\section{DISCUSION Y CONCLUSIONES}

Aunque existe un número importante de yacimientos de vertebrados en el Cretácico Inferior español, un amplio porcentaje de los mismos se conoce únicamente a través de citas antiguas y puntuales, de modo que la comparación que puede establecerse entre dichos yacimientos y el yacimiento de "Zorralbo" no tiene gran interés. Necesariamente la comparación debe realizarse con aquellos yacimientos no puntuales y mejor estudiados, pero no puede ser una comparación exhaustiva en tanto y en cuanto los paleoambientes y las edades de depósito de los mismos son diferentes y lo mismo sucede con los métodos empleados en la excavación, recogida y conservación de sus restos fósiles.

Estas razones nos han llevado a comparar nuestros datos únicamente con los de los siguientes yacimientos, de los que damos una breve reseña bibliográfica ya que son ampliamente conocidos:

Los afloramientos de Galve en Teruel tienen edades comprendidas entre el Jurásico terminal y el Barremiense inferior y se formaron en un ambiente de abanico fluvial con influencia mareal. Se han recuperado restos de dinosaurios saurópodos (entre ellos los géneros Aragosaurus y Pleurocoelus), terópodos, ornitópodos (géneros Iguanodon, Hypsilophodon y Valdosaurus) y tireóforos (cf. Echinodon) En casi todos ellos se ha cribado el sedimento con lo que la lista de vertebrados es muy amplia con diversas especies de pequeños mamíferos, pterosaurios, cocodrilos, saurios, anfibios y peces (Royo y Gómez, 1926a; Lapparent, 1960; Kühne, 1966; Krebs, 1980; Estés \& Sanchiz, 1982; Sanz, 1982; Sanz et al., 1984a, 1984b, 1987; Buscalioni \& Sanz, 1984, 1987; Kohring, 1990; Pérez Oñate et al., 1994; Ruiz-Omeñaca \& Cuenca-Bescós, 1995; Ruiz-Omeñaca, 1996; Canudo et al., 1996a).

El yacimiento de "La Cantalera" en Olite (Teruel) es de edad Barremiense inferior y se originó en un ambiente palustre mientras que el de Vallipón en Castellote (Teruel) pertenece al Barremiense superior y se ha formado en un medio intermareal o submareal. En estos casos se ha pro- 
cedido a la disgregación de la roca con ácido fórmico y se han obtenido, sobre todo, dientes de dinosaurios saurópodos, terópodos y ornitópodos (entre ellos el género Iguanodon) y de pequeños vertebrados. En Vallipón se ha señalado la presencia de mamíferos de pequeño tamaño, pterosaurios, cocodrilos, saurios, plesiosaurios y peces; en "La Cantalera", de tortugas y cocodrilos (Canudo et al., 1996b; Ruiz-Omeñaca et al., 1996, 1997).

El conjunto de yacimientos de Morella (Castellón) se sitúa en el Aptiense inferior, en un medio deltaico con influencia mareal. No han proporcionado restos de pequeños vertebrados pero si de dinosaurios saurópodos, terópodos, ornitópodos (géneros Iguanodon e Hypsilophodon) y tireóforos (género Polacanthus) (Vilanova, 1873; Santafé et al., 1982; Sanz et al., 1983).

Los yacimientos de Valencia en la cuenca de "Los Serranos", en la localidad de La Losilla, han dado los restos de dos importante taxones, un dinosaurio saurópodo, Losillasaurus giganteus y un dinosaurio estegosaurio, Dacentrurus armatus, que se depositaron en un ambiente fluvial y con una edad Cretácico Inferior (Casanovas et al., 1995a, 1995b, 2001).

Los yacimientos de Cuenca ("Las Hoyas" y Uña) pertenecen al Barremiense inferior y se depositaron en un ambiente lacustre sin ningún tipo de influencia marina. Han proporcionado un gran número de macro y microvertebrados: pequeños mamíferos, aves, cocodrilos, tortugas, saurios, anfibios y peces además de restos de dinosaurios saurópodos, terópodos (entre ellos el género Pelecanimimus) y ornitópodos (género Iguanodon) (Buscalioni et al., 1988; Sanz \& Bonaparte, 1992; Sanz \& Buscalioni, 1992; Pérez Moreno et al., 1994; Rauhut \& Zinke, 1995; Sanz \& Pérez Moreno, 1995; Sanz et al., 1995).

Los yacimientos de Burgos están ampliamente distribuidos, los restos se han recogido en superficie excepto en el caso del yacimiento de Ahedo, y su edad va desde el Hauteriviense superior al Aptiense. Se han recuperado restos fósiles de dinosaurios saurópodos (género Rebbachisaurus), terópodos (género Baryonyx), ornitópodos (Hypsilophodon foxii e Iguanodon fittoni) y tireóforos (género Polacanthus y Dacentrurus) además de restos de tortugas (Salasemys pulcherrima) y cocodrilos (Sanz, 1983; Torcida, 1996; Torcida et al., 1997; Maisch, 1997; Fuentes Vidarte \& Meijide Calvo, 1999; Fuentes Vidarte et al., 1999, 2003; Pereda Suberbiola et al., 1999, 2003a, 2003b).

En La Rioja, los yacimientos son escasos y con restos referidos a un solo taxon. En Igea de edad Cretácico inferior han aparecido los restos de un dinosaurio de la especie Hypsilophodon foxii y parte de la mandíbula de un dinosaurio de la especie Baryonyx walkeri, en Vadillos de San Román, de edad Cretácico inferior, restos de un cocodrilo del género Goniopholis y en Préjano, los restos de un grupo de pterosaurios. (Torres \& Viera, 1994; Viera \& Torres, 1995; Ortega et al., 1996; Fuentes Vidarte et al., 1999).
En Soria, se conocen muy pocos yacimientos, el de "Los Caños" del Hauteriviense superior-Barremiense inferior, de ambiente fluvio-lacustre, que no es otra cosa que la continuación del yacimiento de "Zorralbo" y que ha proporcionado restos muy escasos de dinosaurios ornitópodos y terópodos, cocodrilos, tortugas y peces del género Lepidotes; el de Tera, donde apareció un fémur de un dinosaurio saurópodo aún sin determinar, de edad Cretácico Inferior y los de Huérteles y Valduérteles, de edad TitónicoBerriasiense, que han proporcionado restos de pterosaurios sin determinación genérica (Royo y Gómez, 1926d, 1929; Saenz García, 1955; Sanz et al., 1992; Fuentes Vidarte, 1998; Fuentes Vidarte et al., 1999, 2003).

Analizando las listas de fauna de los yacimientos citados, parece claro que hay una serie de taxones que aparecen en casi todos ellos y que demuestran no sólo una amplia distribución territorial y medio-ambiental sino una gran longevidad.

Así por ejemplo, el género Iguanodon está presente en los yacimientos de Soria, Burgos, Cuenca, Teruel y Castellón desde el Hauteriviense superior-Barremiense inferior (-117,5 a -115 m.a) hasta el Aptiense (-112 a -109,5 m.a.), los Nodosáurios se encuentran desde el Hauteriviense superior-Barremiense inferior hasta el Aptiense en Soria, Burgos, Castellón y Teruel y lo mismo sucede con el grupo de los hipsilofodóntidos. Otros vertebrados que aparecen de forma constante son los cocodrilos de tipo Goniopholis y los peces del género Lepidotes. Pero también está claro que, dependiendo del paleoambiente, se origina una gran variación del espectro faunístico, con predominio de tal o cual tipo de vertebrado: peces, anfibios, saurios, tortugas, cocodrilos, pterosaurios, aves, etc y presencia de diversos tipos de dinosaurios, representados mayoritariamente por sus dientes.

Con estos datos, a la única conclusión a la que podemos llegar es que la fauna fósil recogida en "Zorralbo" se corresponde con ese grupo permanente de taxones y que comparando con las listas faunísticas de los yacimientos antes señalados, el más cercano, pese a la diferencia de edad, es el conjunto de yacimientos de Morella, con la salvedad de que en el yacimiento de "Zorralbo", en $150 \mathrm{~m}^{2}$ de extensión, se acumulan todos los taxones citados, con predominio de los vertebrados fitófagos sobre los carnívoros. En los dos yacimientos se han encontrado los restos de un pequeño dinosaurio hipsilofodóntido, los de un sauropodo titanosauriforme (braquiosáurido) y los de un gran terópodo, de una tortuga y de un cocodrilo goniofólido, sin que hasta el momento se haya señalado la existencia de otros vertebrados.

\section{AGRADECIMIENTOS}

Nuestro agradecimiento a la Dirección General de Patrimonio de Castilla y León y al Ayuntamiento de Golmayo por su interés y colaboración. Al Director del Museo Numantino de Soria, Elías Terés por su ayuda desinteresada y colaboración, a 
Luis Giménez, que planteó el sistema de excavación, y en particular a Felix Sanz Aldea, María Pérez Santabárbara y "Cani", compañeros de fatigas así como a todos aquellos amigos que de una manera altruista nos prestaron su ayuda física para sacar adelante la excavación.

\section{BIBLIOGRAFÍA}

Alía Medina, M. 1942. Excursión geológica a Guadalajara, Soria y Logroño. Boletín Real Sociedad española historia Natural, 40, 137-156.

Beuther, A. 1966. Geologische Untersuchungen in Wealden und Utrillas Schichten in Westteil der Sierra de los Cameros (Norddwestliche Iberische Ketten) (Spanien). Beithefte Geologischen Jahrbuch, 55, 103-121.

Brain, C. K. 1981. The Hunters or the Hunted. An introduction to African Cave Taphonomy. The University of Chicago Press, $361 \mathrm{pp}$.

Buscalioni, A. D. 1986. Los cocodrilos fósiles del registro español. Paleontologia i Evolució, 20, 93-98.

Buscalioni, A. D., Barbadillo, L. J. \& Sanz, J. L. 1988. Los reptiles diápsidos de Las Hoyas (Cretácico inferior, Cuenca). IV Jornadas de Paleontología. Resúmenes de las Comunicaciones, Salamanca, 27.

Buscalioni, A. D., Buffetaut, E. \& Sanz, J. L. 1984. An immature specimen of the crocodilian Bernissartia from the Lower Cretaceous of Galve (Province of Teruel, Spain). Palaeontology, 27, 809-813.

Buscalioni, A. D. \& Sanz, J. L. 1984. Los Arcosaurios (Reptilia) del Jurásico superior-Cretácico inferior del area de Galve (Teruel, España). Teruel, 71, 9-28.

Buscalioni, A. D. \& Sanz, J. L. 1987. Lista faunística de los vertebrados de Galve (Teruel). Estudios Geológicos, vol. ext. Galve-Tremp, 65-67.

Canudo, J. I., Cuenca Bescós, G., Ruiz Omeñaca, J. I. \& Soria, A. R. 1996a. Registro fósil de vertebrados en el tránsito Jurásico-Cretácico de Galve (Teruel). Revista de la Academia de Ciencias Zaragoza, 51, 221-236.

Canudo, J. I, Cuenca Bescós, G., Ruiz Omeñaca, J. I. \& Soria, A. R. 1996b. Estratigrafía y paleoecología de los vertebrados del Barremiense superior (Cretácico inferior) de Vallipón (Castellote, Teruel). Mas de las Matas, 15, 9-34.

Casanovas, M. L., Santafé, J. V. \& Pereda Suberbiola, X. 1995a. Presencia por primera vez en España de Dinosaurios estegosaurios (Cretácico inferior de Aldea de Losilla, Valencia). Revista Española de Paleontología, 10, 83-89.

Casanovas, M. L., Santafé, J. V. \& Santisteban Bové. 1995b. Dacentrurus armatus (Stegosauria, Dinosauria) en el Cretácico inferior de la comarca de Los Serranos (Valencia, España). Revista Española de Paleontología, 10, 273-283.

Casanovas, M. L., Santafé, J. V. \& Sanz, J. L. 2001. Losillasurus giganteus, un nuevo saurópodo del tránsito Jurásico-Cretácico de la cuenca de "Los Serranos" (Valencia, España). Paleontología i Evolució, 32-33, 99-122.
Clemente, P. 1988. Sedimentos fluvio-lacustres en la base de la megasecuencia Hauteriviense superior-Aptiense, borde meridional de la cuenca de los Cameros, provincia de Soria. Geogaceta, 5, 19-21.

Clemente, P. \& Alonso, A. 1990. Estratigrafía y sedimentología de las facies continentales del Cretácico inferior en el borde meridional de la cuenca de los Cameros. Estudios Geológicos, 46, 257-276.

Clemente, P. \& Pérez Arlucea, M. 1993. Depositional architecture of the Cuerda del Pozo Formation, Lower Cretaceous of the extensional Cameros Basin, North-Central Spain. Journal of Sedimentary Petrology, 63, 437-452.

Clemente, P., Alonso, A. \& Pérez Arlucea, M. 1991. secuencias de depósito en la parte occidental de la Cuenca de Los Cameros. Jurásico terminal-Cretácico inferior. III Coloquios del Cretácico de España. Morella, Counicaiones, $\mathbf{1}, 21$.

Dollo, M. L. 1885. Cinquème note sur les Dinosauriens de Bernissart. Bulletin Museum royal Histoire Naturlle Belgique, 3, 129-146.

Estés, R. \& Sanchiz, B. 1982. Early Cretaceous Lower Vertebrates from Galve (Teruel), Spain. Journal of Vertebrate Paleontology, 2, 21-39.

Fiorillo, A. R. 1988. Taphonomy of Hazard Homestead Quarry (Ogallala Group), Hitchcock County, Nebraska. Contributions to Geology, University of Wyoming, 26, 57-97.

Fuentes Vidarte, C. 1998. Informe preliminar de la prospección paleontológica del yacimiento de "Los Caños" en Golmayo (Soria 1996-1997). Dirección General de Patrimonio y Promoción Cultural. Junta de Castilla y León, 22 pp. (inédito)

Fuentes Vidarte, C. \& Meijide Calvo, M. 1996. Restos de pterosaurios en el Weald de Soria (España). Studia Geologica Salmanticensia, 32, 15-22.

Fuentes Vidarte, C. \& Meijide Calvo, M. 1999. Presencia de crías de Hypsilophodon foxii (Dinosauria, Ornithopoda) en el Weald de Salas de los Infantes (Burgos, España). Actas I Jornadas Internacionales sobre Paleontología de Dinosaurios Salas de los Infantes (Burgos), 1999, 339-348.

Fuentes Vidarte, C., Meijide Calvo, M., Izquierdo, L. A., Montero, D., Pérez, G., Torcida, F., Urien, V., Meijide Fuentes, F. \& Meijide Fuentes, M. 1999a. Restos fósiles de Baryonyx (Dinosauria, Theropoda) en el Cretácico inferior de Salas de los Infantes (Burgos, España). Actas I Jornadas Internacionales sobre Paleontología de Dinosaurios, Salas de los Infantes (Burgos), 1999, 349-359

Fuentes Vidarte, C., Meijide Calvo, M. \& Torcida, F. 1999b. restos de pterosaurios en Préjano (La Rioja). Memoria-Inventario para el Centro de Estudios Riojanos (inédito).

Fuentes Vidarte, C., Meijide Calvo, M., Meijide Fuentes, M., Meijide Fuentes, F. \& Sanz Aldea, F. 2002. Primeros restos de Iguanodon (Dinosauria, Ornithischia) en el Cretácico inferior de Soria (España). Resúmenes I Congreso Internacional sobre dinosaurios y otros reptiles Mesozoicos de España, Logroño, 17.

Fuentes Vidarte, C., Meijide Calvo, M. \& Meijide Fuentes, F. 2003a. Nueva tortuga para el Cretácico inferior de Salas 
de los Infantes (Burgos, España): Salasemys pulcherrima nv. gen. nv. sp. Studia geologica Salmanticensia, 39, 109-123.

Fuentes Vidarte, C., Meijide Calvo, M., Meijide Fuentes, F. \& Meijide Fuentes, M. 2003b. Fauna fósil del yacimiento Mesozoico (Cretácico Inferior, Wealdense) de "Los Caños" (Soria, España). Celtiberia, 97, 487-506.

Galton, P. M. 1974. The Ornithischian Dinosaur Hypsilophodon foxii from the Wealden of the isle of Wight. Bulletin British Museum (Natural History), Geology, 25, 155 pp.

Kohring, R. 1990. Fossile Reptil-Eischalen (Chelonia, Crocodilia, Dinosauria) aus dem unteren Barremium von Galve (Provinz Teruel, SE-Spanien). Paläontologische Zeitschrift, 64, 329-344.

Krebs, B. 1980. The search for Mesozoic Mammals in Spain and Portugal. Mesozoic Vertebrate Life, 1, 23-25.

Kühne, W. 1966. Decouverte de dents de mammifères dans le Wealdien de Galve (province de Teruel, Espagne). Teruel, 35, 159-161.

Lapparent, A. F. 1960. Los dos dinosaurios de Galve. Teruel, 24, 177-206.

Lapparent, A. F., Quintero, I. \& Trigueros, E. 1957. Descubrimientos de huesos de dinosaurios en el Cretáceo terminal de Cubilla (Provincia de Soria). Notas y Comunicaciones Instituto Geologico Minero de España, 45, 61-64.

Mas, J. R., Alonso, A. \& Guimerá, J. 1993. Evolución tectonosedimentaria de una cuenca extensional intraplaca: la cuenca finijurásica-eocretácica de Los Cameros (La RiojaSoria). Revista Sociedad Geológica España, 6, 129-144.

Maisch, H. W. 1997. The Lower Cretaceous dinosaur Iguanodon cf. fittoni Lydekker 1889 (Orniyhischia) from Salas de los Infantes (Province Burgos, Spain). Neues Jahrbuch fur Geologie und Paläontologie, Mh., 1997, 213-222.

Martín-Closas, C. \& Alonso Millán, A. 1998. Estratigrafía y bioestratigrafía (Charophyta) del Cretácico inferior en el sector occidental de la Cuenca de Cameros (Cordillera Ibérica). Revista Sociedad Geológica España, 11, 253-269.

Meléndez, N. 1978. El Cretácico inferior en la zona de Picofrentes-Las Fraguas (Provincia de Soria). Tesis de Licenciatura U.C.M., 124 pp. (inédita).

Metcalf, S. J. 1993. The palaeoenvironment of a new British dinosaur locality from the Lower Bathonian (Middle Jurassic). Révue de Paléobiologie, vol. esp., 7, 125-149.

Norman, D. B. 1980. On the Ornithischian dinosaur Iguanodon bernissartensis from the Lower Cretaceous of Bernissart (Belgium). Memoire Intitut Royal des Sciences Naturelles de Belgique, 178, 1-93.

Norman, D. B. 1986. On the anatomy of Iguanodon atherfieldensis (Ornithischia: Ornithopoda). Bulletin Institut Royal des Sciences Naturelles de Belgique. Sciences de la Terre, 56, 281-372.

Ortega, F., Moratalla, J. J., Buscalioni, A. D., Sanz, J. L., Jiménez, S. \& Valbuena, J. 1996. Sobre la presencia de un cocodrilo fósil (Crocodylomorpha: Neosuchia, Goniopholis sp.) en la Cuenca de Cameros (Cretácico inferior: Vadillos-San Román de Cameros, La Rioja). Zubía, 14, 113-120.

Palacios, P. 1890. Descripción física, geológica y agrológica de la provincia de Soria. Memorias de la Comisión del Mapa Geológico de España, 550 pp.

Palacios, P. \& Sánchez Lozano, R. 1885. La formación wealdense en las provincias de Soria y Logroño. Boletin de la Comisión del Mapa Geológico de España, 12, 109-140.

Pereda Suberbiola, X. 1994. Polacanthus (Ornithischia, Ankylosauria), a transatlantic armoured dinosaur from the early Cretaceous of Europe and North America. Sonderabdruck aus Palaeontographica, 232, 133-159.

Pereda Suberbiola, X., Meijide, M., Torcida, F., Welle, J., Fuentes, C., Izquierdo, L. A., Montero, D., Pérez, G. \& Urien, V. 1999. Espinas dérmicas del dinosaurio anquilosaurio Polacanthus en las facies Weald de Salas de los Infantes (Burgos, España). Estudios Geológicos, 55, 267-272.

Pereda Suberbiola, X. \& Ruiz Omeñaca, J. I. 2001. Un dinosaurio saurópodo (Titanosauria) en el Cretácico superior de Cubilla, Soria (España). Geogaceta 30, 175-178.

Pereda Suberbiola, X., Torcida, F., Izquierdo, L. I., Huerta, P., Montero, D. \& Pérez, G. 2003. First rebbachisaurid dinosaur (Sauropoda, Diplodocoidea) from the early Cretaceous of Spain: palaeobiogeographical implications. Bulletin Societé Geólogique France,174, 471-479.

Pereda Suberbiola, X., Galton, P. M., Torcida, F., Huerta, P., Izquierdo, L. A., Montero, D., Pérez, G. \& Urien, V. 2003. First stegosaurian dinosaur remains from the early Cretaceous of Burgos (Spain), with a review of Cretaceous stegosaurs. Revista Española de Paleontología, 18, 143-150.

Pérez Moreno, B., Sanz, J. L., Buscalioni, A. D., Moratalla, J. J., Ortega, F. \& Rasskin-Gutman, D. 1994. A unique multitoothed ornithomimosaur dinosaur from the Lower Cretaceous of Spain. Nature, 370, 363-367.

Pérez Oñate, J., Cuenca Bescós, G. \& Sanz, J. L. 1994. Un Nuevo Saurópodo del Jurásico superior de Galve (Teruel). Comunicaciones de las X Jornadas de Paleontología, Madrid, 159-162.

Rauhut, O. W. M. \& Zinke, J. 1995. A description of the barremian dinosaur fauna from Uña with a comparison to that of Las Hoyas. II International Symposium on Litographic Limestones, Lleida-Cuenca (Spain). Extended Abstracts. Universidad Autónoma de Madrid, 123-126.

Royo y Gómez, 1926a. Los vertebrados del Cretácico español de facies wealdica. Boletín Instituto Geológico Minero de España, 47, 169.

Royo y Gómez, 1926b. Restos de vertebrados de facies wealdica en "Los Caños" (Soria). Actas boletín Real Sociedad española Historia Natural, 26, 317.

Royo y Gómez, J. 1926c. Los descubrimientos de reptiles gigantescos en Levante. Boletín Sociedad Castellonense de Cultura, 7,147-162.

Royo y Gómez, J. 1926d. Nuevos vertebrados de la facies Wealdica de "Los Caños" (Soria) y Benageber. Boletin Real Sociedad Española Historia Natural, 26, 317.

Royo y Gómez, J. 1929. Les vertébrés du faciés Wealdien Espagnol. C. R. XIV Congrès Géologique Internacional, 4, 1-6.

Royo-Torres, R. \& Canudo, J. I. 1999. El dinosaurio saurópodo (Aptiense, Cretácico Inferior) de Peñarroya de 
Tastavins (Teruel). Actas I Jornadas Internacionales sobre Paleontología de Dinosaurios Salas de Los Infantes (Burgos) 1999, 417-425.

Ruiz Omeñaca, J. I. 1996. Los dinosaurios hipsilofodóntidos (Reptilia. Ornithischia) del Cretácico Inferior de Galve (Teruel). Tesis de Licenciatura, Universidad de Zaragoza, 338 pp. (inédita).

Ruiz Omeñaca, J. I. \& Cuenca Bescós, G. 1995. Un nuevo dinosaurio hipsilofodóntido (Ornitischia) del Barremiense inferior de Galve (Teruel). XI Jornadas de Paleontología Sociedad Española de Paleontología, 153-156.

Ruiz Omeñaca, J. I., Canudo, J. I. \& Cuenca Bescós, G. 1996. Dientes de dinosaurios (Ornithischia, Saurischia) del Barrremiense superior (Cretácico inferior) de vallipón (Castellote, Teruel). Mas de las Matas, 15, 59-103.

Ruiz-Omeñaca, J. I., Canudo, J. I. \& Cuenca-Bescós, G. 1997. Primera cita de dinosaurios barionícidos (Saurischia, Theropoda) en el Barremiense superior (Cretácico inferior) de Vallipón (Castellote, Teruel). Mas de las Matas, 17, 201-223.

Saenz García, C. 1932. Notas para el estudio de la facies wealdica española. Asociación Española para el progreso de las ciencias (Congreso de Lisboa), sección 4, 59-76.

Saenz García, C. 1955. Nuevos fósiles de vertebrados de "Los Caños" (Soria). Actas Boletín Real Sociedad Española Historia Natural, 53, 21.

Salgado, L., Coria, R. A. \& Calvo, J. O. 1997. Evolution of titanosaurid sauropods. I. Phylogenetic análisis base don the postcranial evidence. Ameghiniana, 34, 3-32.

Santafé, J. V., Casanovas, M. L., Sanz, J. L. \& Calzada, S. 1982. Geología y Paleontología (Dinosaurios) de las Capas rojas de Morella (Castellón, España). Diputación Provincial de Castellón - Diputación de Barcelona, 169 pp.

Sanz, J. L. 1982. A Sauropod dinosaur tooth from the Lower Cretaceous of Galve (Province of Teruel, Spain). Geobios, 15, 93-94.

Sanz, J. L. 1983. A nodosaur ankylosaur from the Lower Cretaceous of Salas de los Infantes (Province of Burgos, Spain). Geobios, 16, 615-621.

Sanz, J. L. \& Bonaparte, J. F. 1992. A new order of birds (class Aves) from the Lower Cretaceous of Spain. Natural History Museum of Los Angeles Country, 36, 39-49.

Sanz, J. L. \& Buscalioni, A. D. 1992. A new bird from the Early Cretaceous of Las Hoyas, Spain, and the early radiations of birds. Palaeontology, 35, 829-845.

Sanz, J. L. \& Moratalla, J. 1993. Dinosaurios en Soria. Revista Soria, segunda época, 2, 3-10.

Sanz, J. L. \& Pérez Moreno, B. 1995. Ornitomimosaurio hallado en Cuenca. Investigación y Ciencia, 221, 31-33.

Sanz, J. L., Santafé, J. V. \& Casanovas, M. L. 1983. Wealden ornitopod dinosaur Hypsilophodon from the capas Rojas formation (Lower Aptian, Lower Cretaceous) of Morella, Castellón, Spain. Journal Vertebrate Paleontology 3, 39-42.

Sanz, J. L., Casanovas, M. L. \& Santafé, J. V. 1984a. Iguanodóntidos (REPTILIA, ORNITOPODA) del yacimiento del Cretácico inferior de San Cristóbal (Galve, Teruel). Acta Geológica Hispánica, 19, 171-176.
Sanz, J. L., Casanovas, M. L. \& Santafé, J. V. 1984b. Restos autopodiales de Iguanodon (Reptilia, Ornithopoda) del yacimiento de Santa Bárbara (Cretácico inferior, Galve, provincial de Teruel, España). Estudios Geológicos, 40, 251-257.

Sanz, J. L., Buscalioni, A. D, Casanovas, M. L. \& Santafé, J. V. 1987. Dinosaurios del Cretácico inferior de Galve (Teruel, España). Estudios Geológicos, vol. ext. GalveTremp, 45-64.

Sanz, J. L., Buscalioni, A. D., Pérez Moreno, B. P., Moratalla, J. J. \& Jiménez García, S. 1992. Los Dinosaurios de Castilla y León. In: Vertebrados fósiles de Castilla y León (Ed. Jiménez Fuentes). Museo de Salamanca, 47-57.

Sanz, J. L., Chiappe, L. M. \& Buscalioni, A. D. 1995. The Osteology of Concornis lacustris (Aves: Enantiornithes) from the Lower Cretaceous of Spain and a Reexamination of its Phylogenetic Relationships. American Museum Novitates, 3133, 1-23.

Taquet, P. 1976. Géologie et paléontologie du gisement de Gadoufaoua (Aptien du Níger). Cahiers de Paléontologie, Centre Nacional de la Recherche Scientifique, Paris, 191 pp.

Tischer, G. 1966. El delta weáldico de las montañas ibéricas occidentales y sus enlaces tectónicos. Notas y Comunicaciones del Instituto Geológico y Minero de España, 81, 53-78.

Torcida, F. 1996. Registro de dinosaurios en el sureste de la provincia de Burgos. Zubía, 14, 89-104.

Torcida, F., Fuentes, C., Izquierdo, L. A., Montero, D. \& Urien, V. 1997. Dientes de dinosaurios terópodos (cf. Baryonyx) en el Weald de Burgos (España). Studia Geológica Salmanticensia, 33, 59-65.

Torcida, F., Pereda Suberbiola, X., Huerta Hurtado, P., Izquierdo, L. A., Montero, D. \& Pérez, G. 2001.- Descripción preliminar de un dinosaurio rebaquisaúrido (Sauropoda, Diplodocoidea) del Cretácico Inferior de Burgos (España). Actas II Jornadas Internacionales sobre Paleontología de Dinosaurios Salas de los Infantes (Burgos) 2001, 203-211.

Torres, J. A. \& Viera, L. I. 1994. Hypsilophodon foxii (Reptilia, Ornithischia) en el Cretácico inferior de Igea (La Rioja, España). Munibe (Ciencias Naturales), 46, 3-41.

Viera, L. I. \& Torres, J. A. 1995. Presencia de Baryonyx walkeri (Saurischia, Theropoda) en el Weald de La Rioja (España). Nota previa. Munibe (Ciencias Naturales), 47, 57-61.

Vilanova, J. 1873. Restos de Iguanodon de los lignitos de Utrillas y otro en Morella. Actas Sociedad Española de Historia Natural, 2, 8.

Weishampel, D. B. \& Horner, J. R. 1992. "Hadrosauridae". In: The Dinosaurio (eds. D. B. Weishampel, P. Dodson \& H. Osmólska). University of California Press, 534-561.

Wilson, J. A. \& Sereno, P. C. 1998. Early evolution and higher-level phylogeny of sauropod dinosaurs. Journal of vertebrate paleontology, 18, 1-68.

Manuscrito recibido: 7 de Diciembre, 2003 Manuscrito aceptado: 17 de Mayo, 2005 